\title{
Linear Parameter-Varying Control of A Power-Synchronized Grid-Following PLL-Less Inverter
}

\author{
Milad Zarif Mansour, Student Member, IEEE, Alireza Karimi, Member, IEEE, Behrooz Bahrani, Senior Member, IEEE
}

\begin{abstract}
This paper proposes a Linear Parameter-Varying loopshaping controller for a power-synchronized Phase-locked Loop (PLL)-less grid-following inverter (GFLI). This control strategy regulates the inverter's output active and reactive powers at the terminal instead of the point of connection and does not require a PLL for extracting the voltage phase angle. Hence, the prevalent stability issues exhibited when GFLIs are connected to weak grids are not present, and the proposed GFLI control strategy can work under both very weak and strong grid conditions without being prone to instability. In this approach, the controller parameters are functions of the operating point and changed during the real-time operation such that the closed-loop performance is preserved in all operating points. Furthermore, since the grid impedance is a factor in the design process, a robustness analysis against grid impedance estimation error is conducted, and it is shown that discrepancies in estimated and real grid impedances are unlikely to make the system unstable. The performance of the proposed control design is validated in Matlab/PLECS and experiment for both strong and weak grids.
\end{abstract}

Index Terms-Linear Parameter-Varying, Loop-Shaping, PhaseLocked Loop, Power Control, Power Synchronized.

\section{INTRODUCTION}

I $\mathrm{N}$ recent years, inverter-based resources (IBRs) have gained increasing importance in power systems as a result of their decreasing cost and increasing concerns regarding $\mathrm{CO}_{2}$ emission. Hence, their penetration in power grids is rapidly increasing, and conventional synchronous generators are being replaced by them. Despite their advantages, IBRs may face stability and grid synchronization issues due to the shortcomings of the conventional control structures used for this purpose [1].

According to their synchronization strategy, IBRs can be classified in two main groups: 1) grid-following inverters (GFLIs) and 2) grid-forming inverters (GFMIs). GFLIs employ Phase-Locked Loops (PLLs) for estimating the grid voltage angle, by which they can get synchronized to the grid [2]-[4]. This type of IBRs uses the point of connection ( $\mathrm{PoC}$ ) voltage to extract the grid voltage angle that is used in a vector current controller to create the gating signals required for the inverter [5]-[7]. GFMIs, on the other hand, adjust the phase angle of the PoC voltage by controlling the inverter's output active power [8]. In this type of inverters, active power-frequency droop control is used to synchronize the inverter with the grid [3], [9]-[11]. Also, the PoC voltage's magnitude is regulated by the inverter based on the output reactive powers, in contrast to GFLIs. In light of different control strategies that are used in these two types of inverters, the stability issues that they may have are different.

As the most prevalent IBR control strategy, GFLIs encounter stability issues while integrated into weak grids [12]. Different studies show the main instability motivation is particularly due to the PLL's issues in weak grids, which might not have an equilibrium point or have an unstable one [13]-[19]. On the other hand, GFMIs face issues when connected to strong grids as it is challenging to regulate the PoC voltage in such grids [20], [21]. Since GFMIs can be modeled as a first-order system, their transient stability improves compared with GFLIs [22]. Despite that, the droop control loop used for reactive power control can negatively affect the transient stability [23]. In addition, GFMIs can induce oscillations while connected to strong or series compensated grids [21], [24], [25]. Moreover, output current limiting during a fault occurrence is challenging for this type of IBRs [26].

To overcome the stability issues corresponding to the GFLIs and GFMIs, some studies proposed direct power control (DPC) for the IBRs that control the IBRs output active and reactive power directly without having an inner control loop [27], [28]. These methods use a variable switching frequency that causes a wide range of harmonics, and hence, it is not easy to design a filter for them. To conquer this, other DPC methods are proposed that have a constant switching frequency [29]-[33]. Nonetheless, these methods could not eliminate power oscillations either, entirely.

A PLL-less voltage-modulated direct power control (VMDPC) for IBRs is introduced in [34]-[36]. Given the fact that the PLL is eliminated from this control structure, the VMDPC does not have the PLL-related issues that conventional GFLIs suffer from. Nonetheless, this method faces problems while integrated into weak grids due to the necessity of PoC voltage sensing [36]. Also, [37] shows that this approach does not possess any merit compared with conventional PLL-based GFLIs provided the PLL is tuned properly. To address the shortcomings of the previous methods for controlling grid-following IBRs, [38] proposes a power-synchronized control strategy. This method, similar to the VMDPC, does not use a PLL for the grid synchronization. However, unlike the VMDPC, since this method does not require PoC voltage measurement, it is not affected in weak-grid conditions, and thus, can perform in both stiff and weak grids. This method uses the inverter's terminal voltage instead of the PoC voltage to control the inverter's terminal output power. This control structure is composed of an outer loop that is responsible for controlling the IBR's active and reactive powers, and generates the grid frequency and inner loop current references. The current controller employed in this control structure is the conventional vector current controller. Although this method is effective, and the IBR can work under stiff and weak grid conditions, the active and reactive power control design method proposed in [38] is not straightforward since it uses optimization techniques for control design. Also, the performance depends on the operating point of the system, and the IBR cannot work as a rectifier since the gain margin of the system becomes negative while working as a rectifier unless the controller is modified.

This paper aims to rectify the shortcomings that [38] has in terms of control design. To do so, a Linear Parameter-Varying (LPV) loop-shaping controller is designed for the proposed IBR control structure. Linear Parameter-Varying systems are a class of nonlinear systems that have the structure of a linear system whose parameters are a function of some scheduling variables that can be measured in real-time operation [39]. A nonlinear system which is linearized around some operating points can be represented as an LPV system in which the scheduling variables are the operating point parameters. The LPV systems are linear time-invariant for fixed scheduling variables. An LPV controller is designed such that 
its parameters are adapted automatically in real-time according to the variation of the operating points. The main benefits of the proposed control design introduced in this paper are

- constant rise time for different operating points,

- straightforward control design based on the IBR operating point, and

- inversion and rectification capabilities.

Also, this study provides a robustness analysis of the proposed controller against the grid impedance estimation error. It is shown that while the order of the system increases in case the grid impedance is not accurately estimated, the system instability is unlikely.

The rest of this paper is organized as follows. Section II discusses the VMDPC and power-synchronized PLL-less gridfollowing inverters. Section III describes the proposed LPV loopshaping control design and analyzes the robustness of the system with respect to discrepancies between the grid's estimated and real impedances. The performance of the proposed method is evaluated in Section IV. Finally, the conclusions are presented in Section V.

\section{GRID INTEGRATION OF INVERTERS}

To have a system that can work in weak and strong grids, a novel power-synchronized control strategy for GFLIs is proposed in [38]. The block diagram of this control strategy is shown in Fig. 1. The advantage of this control strategy compared with conventional GFLIs is in this method, the PLL is not used, and hence, the related stability issues in weak grids is not exhibited. Additionally, this control strategy does not relay on sensing/controlling PoC voltage. Thus, the stability deterioration related to the VMDPC reported in [36], which is due to the affected PoC voltage, is not present in this method. Ref. [38] shows while this method can work in very weak grids, the VMDPC fails; unless the time constant of the system is decreased significantly, which makes the system much slower.

Ref. [38] assumes that the rotating reference frame is aligned with the inverter's output current, which makes $i_{\mathrm{q}}=0 \mathrm{~A}$. Thus, the inverter's output current and voltage can be written as

$$
i_{\mathrm{dq}}=I_{\mathrm{d}}+j 0 \text { and } v_{t, \mathrm{dq}}=V_{t} \cos \delta+j V_{t} \sin \delta .
$$

In (1), $\delta$ is the angle between the inverter output voltage and current, $I_{\mathrm{d}}$ is the d-component of the inverter output current, and $V_{t}$ is the amplitude of the inverter output phase-to-neutral voltage. Also, the inverter's complex output power is

$$
P+j Q=\frac{3}{2}\left(V_{t} I_{\mathrm{d}} \cos \delta+j V_{t} I_{\mathrm{d}} \sin \delta\right)
$$

Supposing that there are small perturbations in $\delta$ and $I_{\mathrm{d}}$, i.e., $\delta=$ $\delta_{0}+\Delta \delta$ and $I_{\mathrm{d}}=I_{\mathrm{d}, 0}+\Delta I_{\mathrm{d}}$, the small-signal model of the system can be derived as [38]

$$
\left(\begin{array}{c}
\Delta P \\
\Delta Q
\end{array}\right)=\underbrace{\left(\begin{array}{cc}
\frac{3 V_{t} I_{\mathrm{d}, 0} \sin \delta_{0}}{2 s} H & \frac{3 V_{t} \cos \delta_{0}}{2(\tau s+1)} H \\
-\frac{3 V_{t} I_{\mathrm{d}, 0} \cos \delta_{0}}{2 s} H & \frac{3 V_{t} \sin \delta_{0}}{2(\tau s+1)} H
\end{array}\right)}_{\mathbf{G}}\left(\begin{array}{c}
\Delta \omega \\
\Delta I_{\mathrm{d}, \mathrm{ref}}
\end{array}\right),
$$

in which $\mathbf{G}$ is the system's transfer function matrix, $H$ is the input low-pass filter and will be neglected from now on, $\frac{1}{\tau s+1}$ represents the current controller assuming that the grid impedance is known, $P$ and $Q$ are inverter output active and reactive power, $\delta_{0}$ is the angle between inverter voltage and current at the operating point, $I_{\mathrm{d}, 0}$ is the inverter output current's d-component, $\omega$ is the angular frequency of the grid estimated by the IBR, and finally, $I_{\mathrm{d} \text {, ref }}$ is the inverter output current's d-component reference.

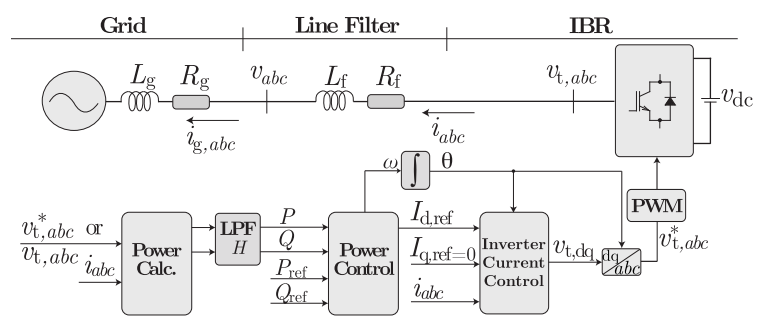

Fig. 1. Power-synchronized GFLI and its control structure.

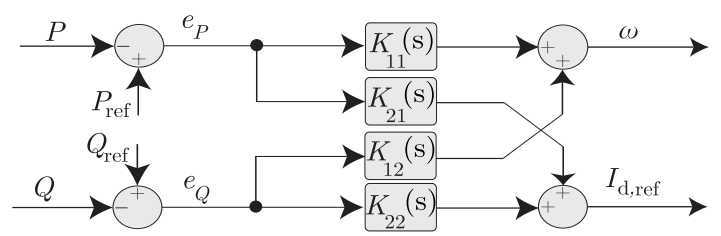

Fig. 2. The block diagram of the proposed power controller.

TABLE I

THE PARAMETERS OF THE STUDY SYSTEM OF FIG. 1.

\begin{tabular}{c|c|c}
\hline \hline Quantity & Value & Comment \\
\hline \hline$L_{\mathrm{f}}$ & $95 \mu \mathrm{H}$ & Inverter Filter Inductance \\
$R_{\mathrm{f}}$ & $0.01 \Omega$ & Series Resistance of $L_{\mathrm{f}}$ \\
\hline$S_{\text {base }}$ & $5 \mathrm{MVA}$ & Inverter Rated Power \\
$v_{\mathrm{g}}$ & $690 \mathrm{~V}$ & Grid Line-to-Neutral Voltage (rms) \\
$v_{\mathrm{dc}}$ & $3000 \mathrm{~V}$ & DC Bus Voltage \\
\hline$f_{\mathrm{sw}}$ & $5 \mathrm{kHz}$ & PWM Carrier Frequency \\
$f$ & $50 \mathrm{~Hz}$ & System Nominal Frequency \\
$f_{\text {filt }}$ & $200 \mathrm{~Hz}$ & Power Measurement LPF Bandwidth \\
\hline
\end{tabular}

An outer-loop control schematic for regulating the output $P$ and $Q$ is proposed in [38], which is shown in Fig. 2. In this figure, $K_{11}(s)$ through $K_{22}(s)$ are Proportional-Integral (PI) compensators. Although the control strategy proposed in [38] is capable of working in very weak and very strong systems, its control design method is complex. First, it uses optimization techniques to design the active and reactive power PI controllers. Additionally, using the PI controllers proposed in [38], the system cannot work as a rectifier since the gain margin of the system becomes negative, making the system unstable. Finally, using the control design method in [38], the bandwidth of the system depends on the operating point of the system, i.e., unless the system operating point is about the operating point the controller is designed for, the system becomes slower or faster than the desired.

Hence, in the following, an alternative controller that addresses the aforementioned shortcomings of the proposed controller in [38] is devised, which is easy to design, has a fixed time constant in different operating points, and can work as an inverter and rectifier.

\section{LPV LOOP-SHAPING CONTROL DESIGN}

As it is seen from (3), the system under study is a MIMO system; hence, a $2 \times 2$ controller must be designed for the system to guarantee stability. To do so, in this study, an LPV loopshaping controller is designed. This controller is designed such that it addresses the drawbacks of the controller designed in [38]. To design the LPV controller, a vector of scheduling parameters should be chosen, which is selected as $\mu=\left[I_{\mathrm{d}, 0}, \delta_{0}\right]^{T}$. Hence, the designed controller and the system both are functions of the vector $\mu$.

Supposing that the desired open-loop transfer function matrix of the system, $\mathbf{L}_{\mathbf{D}}$, is chosen as

$$
\mathbf{L}_{\mathbf{D}}=\left(\begin{array}{cc}
k_{p} / s & 0 \\
0 & k_{p} / s
\end{array}\right)
$$


and assuming that $\mathbf{K}(\mu)$ is the designed $2 \times 2$ controller, the openloop transfer function can be written as

$$
\mathbf{L}(\mu)=\mathbf{G}(\mu) \times \mathbf{K}(\mu) .
$$

In case the open-loop transfer function is equal to the desired transfer function introduced in (4),

$$
\mathbf{K}(\mu)=\mathbf{G}^{-1}(\mu) \times \mathbf{L}_{\mathbf{D}}
$$

Note that the chosen $\mathbf{L}_{\mathbf{D}}$ guarantees stability since the closed-loop transfer functions on the diagonal entries become $\frac{K_{p}}{s+K_{p}}$, which is a stable transfer function. Additionally, $\mathbf{L}_{\mathbf{D}}$ provides a decoupled system as its off-diagonal elements are zero. To find the controller matrix, $\mathbf{K}(\mu)$, the inverse of $\mathbf{G}(\mu)$ can be derived as

$$
\mathbf{G}^{-1}(\mu)=\left(\begin{array}{cc}
\frac{2 s \sin \delta_{0}}{3 V_{t} I_{\mathrm{d}, 0}} & -\frac{2 s \cos \delta_{0}}{3 V_{t} I_{\mathrm{d}, 0}} \\
\frac{2(\tau s+1) \cos \delta_{0}}{3 V_{t}} & \frac{2(\tau s+1) \sin \delta_{0}}{3 V_{t}}
\end{array}\right) .
$$

By replacing (7) in (6), the controller matrix becomes

$$
\mathbf{K}(\mu)=\left(\begin{array}{cc}
k_{p} \frac{2 \sin \delta_{0}}{3 V_{t} I_{\mathrm{d}, 0}} & -k_{p} \frac{2 \cos \delta_{0}}{3 V_{t} I_{\mathrm{d}, 0}} \\
k_{p} \frac{2(\tau s+1) \cos \delta_{0}}{3 V_{t} s} & k_{p} \frac{2(\tau s+1) \sin \delta_{0}}{3 V_{t} s}
\end{array}\right) .
$$

Keeping in mind that $i_{\mathrm{q}}=0 \mathrm{~A}$, the apparent power can be written as

$$
S=\frac{3}{2} V_{t} I_{\mathrm{d}}
$$

By replacing (9) in (8), the designed controller can be reformulated as

$$
\mathbf{K}(\mu)=\left(\begin{array}{cc}
k_{p} \frac{\sin \delta_{0}}{S_{0}} & -k_{p} \frac{\cos \delta_{0}}{S_{0}} \\
k_{p} \frac{(\tau s+1) I_{\mathrm{d}, 0} \cos \delta_{0}}{s \times S_{0}} & k_{p} \frac{(\tau s+1) I_{\mathrm{d}, 0} \sin \delta_{0}}{s \times S_{0}}
\end{array}\right),
$$

in which $S_{0}$ is the inverter apparent power at the operating point and can be written as $S_{0}=\sqrt{P_{\text {ref }}^{2}+Q_{\text {ref }}^{2}}$. Additionally, $\delta_{0}=\tan ^{-1} \frac{Q_{\text {ref }}}{P_{\text {ref }}}$, and $I_{\mathrm{d}, 0}$ is found via load-flow equations at steady state. Using these values, the gains of the controller shown in (10) can be adapted to the new operating point of the system upon each change in the set-points. However, the scheduling parameters should match the real values in the circuit during the transients as well. Since the system's operating point changes with a rise time adjusted by $k_{p}$, the scheduling parameters can be estimated by applying a filter with a similar time constant, i.e., $\frac{1}{T s+1}$, to $P_{\text {ref }}$ and $Q_{\text {ref }}$ used for adjusting the coefficients in (10) during the transients.

The main advantage of the controller proposed in (10) compared to the control design method in [38] is that (10) is easy to design and does not require system identification and/or solving an optimization-based loop-shaping problem since it provides a parametric loop-shaping controller. Also, unlike the method proposed in [38], (10) schedules its gains based on the operating point of the system. Hence, in contrast to [38], the bandwidth of the system is not changed by changing the IBR's operating point. Finally, this controller allows the system to perform bidirectionally, i.e., in inverter and rectifier modes. However, the controller designed in [38] does not work in rectifier mode.

As mentioned above, the transfer function in (3) assumes that the grid impedance is known and the current controller can be simplified to a first-order system with a time constant of $\tau$. This transfer function is used to design the controller proposed in (8). Although there are many approaches for impedance estimation in the literature [40], [41], errors in the grid impedance estimation can occur in the system; thus, a robustness analysis of the system against impedance estimation errors is needed.

\section{A. Robustness Analysis Against Grid Impedance Estimation Error}

Supposing that the grid inductance and resistance are known, the PI controller employed in the current controller can be devised such that it has a zero at $L s+R$, where $L$ is the grid inductance, and $R$ is the grid resistance; hence, the open-loop transfer function of the current controller can be written as $\frac{K_{p, \mathrm{cc}}}{s+K_{p, \mathrm{cc}}}$, or alternatively, $\frac{1}{\tau_{1} s+1}$, where $\frac{1}{K_{p, \mathrm{cc}}}=\tau_{1}$. If the exact values of the grid impedance and resistance are not known, the open-loop transfer function corresponding to the current controller becomes

$$
G_{o, \mathrm{cc}}(s)=K_{p, \mathrm{cc}} \times \frac{\hat{L} s+\hat{R}}{s} \times \frac{1}{L s+R},
$$

in which $\hat{L}$ and $\hat{R}$ are the estimated grid inductance and resistance, respectively. In this study, it is supposed that the resistance and inductance estimation error percentages are the same, i.e., $\hat{L}=$ $a \times L$ and $\hat{R}=a \times R$, in which $a$ denotes the estimation error. With this assumption, the open-loop zero and pole in (11) still cancel each other; however, the estimation error is reflected in the open-loop gain and makes it $a \times K_{p, c c}$. Supposing $\frac{1}{a \times K_{p, c c}}=\tau_{2}$, the current controller closed-loop transfer function can be written as

$$
G_{\mathrm{cc}}(s)=\frac{1}{\tau_{2} s+1} .
$$

Replacing (12) in (3) results in

$$
\mathbf{G}(\mu)=\left(\begin{array}{cc}
\frac{3 V_{t} I_{\mathrm{d}, 0} \sin \delta_{0}}{2 s} & \frac{3 V_{t} \cos \delta_{0}}{2\left(\tau_{2} s+1\right)} \\
-\frac{3 V_{t} I_{\mathrm{d}, 0} \cos \delta_{0}}{2 s} & \frac{3 V_{t} \sin \delta_{0}}{2\left(\tau_{2} s+1\right)}
\end{array}\right) .
$$

By replacing (13) in (5), the open-loop transfer function of the system with the estimation error can be written as

$$
\begin{aligned}
\mathbf{L}(\mu) & =\mathbf{G}(\mu) \times \mathbf{K}(\mu)=\left(\begin{array}{cc}
\frac{k_{1}}{s}+\frac{k_{2}}{s} \times \frac{\tau_{1} s+1}{\tau_{2} s+1} & -\frac{k_{3}}{s}+\frac{k_{3}}{s} \times \frac{\tau_{1} s+1}{\tau_{2} s+1} \\
\frac{k_{3}}{s}-\frac{k_{3}}{s} \times \frac{\tau_{1} s+1}{\tau_{2} s+1} & \frac{k_{2}}{s}+\frac{k_{1}}{s} \times \frac{\tau_{1} s+1}{\tau_{2} s+1}
\end{array}\right) \\
& =\left(\begin{array}{cc}
\frac{k_{1}\left(\tau_{2} s+1\right)+k_{2}\left(\tau_{1} s+1\right)}{s\left(\tau_{2} s+1\right)} & \frac{-k_{3}\left(\tau_{2} s+1\right)+k_{3}\left(\tau_{1} s+1\right)}{s\left(\tau_{2} s+1\right)} \\
\frac{k_{3}\left(\tau_{2} s+1\right)-k_{3}\left(\tau_{1} s+1\right)}{s\left(\tau_{2} s+1\right)} & \frac{k_{2}\left(\tau_{2} s+1\right)+k_{1}\left(\tau_{1} s+1\right)}{s\left(\tau_{2} s+1\right)}
\end{array}\right)
\end{aligned}
$$

in which

$$
k_{1}=k_{p} \sin ^{2} \delta_{0} \quad ; \quad k_{2}=k_{p} \cos ^{2} \delta_{0} \quad ; \quad k_{3}=k_{p} \frac{\sin 2 \delta_{0}}{2} .
$$

In order to evaluate the stability of the system, the poles of the system's closed-loop transfer function must be found. To do so, the closed-loop transfer function can be written as

$$
\mathbf{G}_{\mathbf{c l}}(\mu)=\mathbf{L}(\mu) \times(\mathbf{L}(\mu)+\mathbf{I})^{-1} .
$$

To find the closed-loop system's poles, the characteristic equation of each entry in (16) should be found. Supposing that the system is minimal (no pole-zero cancellation occurs in the system), it can be shown that each entry's characteristic equation, $\Delta(s)$, is the numerator of $\operatorname{det}(\mathbf{L}(\mu)+\mathbf{I})$. Hence, to have a stable system, the roots of $\Delta(s)$ should be negative. The characteristic equation can be found as

$$
\begin{aligned}
\Delta(s) & =\overbrace{\left(k_{1} k_{2}+k_{3}^{2}\right)}^{A}\left(\tau_{2} s+1\right)^{2}+\overbrace{\left(k_{1} k_{2}+k_{3}^{2}\right)}^{A}\left(\tau_{1} s+1\right)^{2} \\
& +\overbrace{\left(k_{1}^{2}+k_{2}^{2}-2 k_{3}^{2}\right)}^{B}\left(\tau_{1} s+1\right)\left(\tau_{2} s+1\right) \\
& +\overbrace{\left(k_{1}+k_{2}\right)}^{C} s\left(\tau_{2} s+1\right) \\
& +\overbrace{\left(k_{1}+k_{2}\right)}^{C} s\left(\tau_{1} s+1\right)\left(\tau_{2} s+1\right)+s^{2}\left(\tau_{2} s+1\right) .
\end{aligned}
$$


Thus, $\Delta(s)$ can be re-written as

$$
\begin{aligned}
\Delta(s) & =\tau_{2}^{2} s^{4}+\left(2 \tau_{2}+C \tau_{1} \tau_{2}\right) s^{3} \\
& +\left\{A\left(\tau_{2}^{2}+\tau_{1}^{2}\right)+(B+C)\left(\tau_{1}+\tau_{2}\right)+C \tau_{2}+1\right\} s^{2} \\
& +\left\{2 A\left(\tau_{1}+\tau_{2}\right)+B\left(\tau_{1}+\tau_{2}\right)+2 C\right\} s+2 A+B .
\end{aligned}
$$

To have a stable system, the roots of (18) must be negative. To find the stability condition, the Routh-Hurwitz criterion is employed. Based on this criterion, to have a stable system,

$$
\begin{aligned}
& \text { 1) } 2 A+B>0, \\
& \text { 2) } 2 \tau_{2}+C \tau_{1} \tau_{2}>0, \text { and } \\
& \text { 3) }\left\{\left(2 \tau_{2}+C \tau_{1} \tau_{2}\right)\left[A\left(\tau_{1}^{2}+\tau_{2}^{2}\right)+(B+C)\left(\tau_{1}+\tau_{2}\right)+C \tau_{2}+1\right]\right. \\
& \left.-\tau_{2}^{2}\left[2 A\left(\tau_{1}+\tau_{2}\right)+B\left(\tau_{1}+\tau_{2}\right)+2 C\right]\right\} \\
& \times\left\{2 A\left(\tau_{1}+\tau_{2}\right)+B\left(\tau_{1}+\tau_{2}\right)+2 C\right\} \\
& -\left(2 \tau_{2}+C \tau_{1} \tau_{2}\right)^{2}(2 A+B)>0 .
\end{aligned}
$$

The first condition in (19) is always valid since $2 A+B=\left(k_{1}+\right.$ $\left.k_{2}\right)^{2}$. Also, since $C=k_{1}+k_{2}>0$, and considering that $\tau_{1}$ and $\tau_{2}$ are positive values, the second condition in (19) is always valid. To evaluate the last condition, it should be noted that the current controller's time constant $(\tau)$ is about a couple of ms, hence, the terms containing higher order of $\tau$ s, i.e., their multiplications or higher powers, can be neglected. By doing so, the third condition can be simplified to $4 C \tau_{2}$, which is always positive.

Therefore, it can be concluded that if the inductance and resistance estimation error percentages are the same, the system will remain stable, while some dynamics are added to the system.

In a general case, when the inductance and resistance estimations have random and not necessarily equal errors, the pole and zero in (11) do not cancel each other, and hence, neglecting the coupling between current's $\mathrm{d}$ and q-components, the current controller closed-loop transfer function becomes

$$
G_{\mathrm{cc}_{\text {general }}}(s)=K_{p, \mathrm{cc}} \frac{\hat{L} s+\hat{R}}{L s^{2}+\left(R+K_{p, \mathrm{cc}} \hat{L}\right) s+K_{p, \mathrm{cc}} \hat{R}},
$$

and the system's transfer function becomes

$$
\mathbf{G}(\mu)=\left(\begin{array}{cc}
\frac{3 V_{t} I_{\mathrm{d}, 0} \sin \delta_{0}}{2 s} & \frac{3 V_{t} \cos \delta_{0}}{2} G_{\mathrm{cc}_{\text {general }}}(s) \\
-\frac{3 V_{t} I_{\mathrm{d}, 0} \cos \delta_{0}}{2 s} & \frac{3 V_{t} \sin \delta_{0}}{2} G_{\mathrm{cc}_{\text {general }}}(s)
\end{array}\right) .
$$

In this study, a numerical robustness analysis of the system introduced in Table I with the transfer function shown in (21) and the controller shown in (10) is done. In this analysis, it is assumed that the resistance estimation error is $10 \%$, and the inductance estimation error varies from $5 \%$ to $95 \%$ with $5 \%$ steps. As discussed, the closed-loop system's poles are the characteristic equation's roots, $\Delta(s)$. Therefore, to assess the stability, the $\Delta(s)$ 's roots are shown in Fig. 3. The arrows show the estimation error increase. It is seen that the overall closed-loop system remains stable despite different estimation errors resistance and inductance have.

It should be noted that the presence of error in the inductance and resistance estimations adds more poles and zeros to the closed-loop transfer function, and hence, some new dynamics will be added to the system; nonetheless, it is not likely that these estimation errors threat the system's stability.

\section{Performance Evaluation}

To assess the performance of the proposed controller, the system shown in Fig. 1 is simulated in Matlab/PLECS. Also,

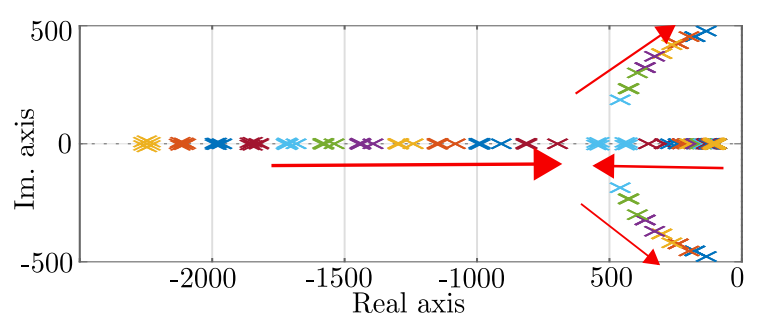

Fig. 3. The characteristic equation's roots when the resistance estimation error is $10 \%$ and the inductance estimation error varies from $5 \%$ to $95 \%$.

the performance of the designed controller is validated using an experimental setup based on Imperix B-Box Controller and Regatron AC Power Supply. In the simulation tests, the ability of the controller for coping with active and reactive power setpoint changes, bi-directional performance, phase jump, and fault occurrence is validated under weak and strong grid conditions. In addition, the experimental setup is employed to validate the ability of the controller to cope with active and reactive power set-point changes.

\section{A. Simulation Results}

To evaluate the performance of the proposed controller, different tests are conducted in Matlab/PLECS when the system shown in Fig. 1 and with the parameters in Table $I$ is subjected to faults, grid phase jumps, and set-point changes both for strong and weak grids.

1) Strong grid $(S C R=17)$ : In this part, the grid inductance and resistance are chosen as $L_{\mathrm{g}}=51 \mu H$ and $R_{\mathrm{g}}=5.4 \mathrm{~m} \Omega$, respectively, making $\mathrm{SCR}=17$. Also, the PI controller employed in the current controller is $2500 \times \frac{146 \times 10^{-6} s+0.0154}{s}$, making the current controller a first-order system with $\tau=0.0004 \mathrm{~s}$. Besides, the $k_{p}$ is chosen equal to 100 , causing a $10 \mathrm{~ms}$ overall time constant. Initially, the converter injects $1 \mathrm{MW}$ and $1 \mathrm{MVAR}$ of active and reactive power, respectively. At $t=0.05 \mathrm{~s}$, the active power set-point is changed to $2 \mathrm{MW}$. At $t=0.15 \mathrm{~s}$, the reactive power set-point steps up to $4 \mathrm{MVAR}$, while the active power remains unchanged. At $t=0.25 \mathrm{~s}$, the power factor is changed from 0.44 to 0.9 , changing the active and reactive power setpoints to $4 \mathrm{MW}$ and $2 \mathrm{MVAR}$, respectively. Finally, at $t=0.35 \mathrm{~s}$, the converter's active power set-point is changed to $-2 \mathrm{MW}$, making the converter to work as a rectifier. Fig. 4 depicts the simulation results of this system. Fig. 4 (a) shows the three-phase output current, Fig. 4 (b) is the converter's filter's output line-toneutral three-phase voltage, Fig. 4 (c) is d and q-components of the converter's output current, Fig. 4 (d) is d and q-components of the converter's terminal voltage, Fig. 4 (e) depicts the output active and reactive power, and finally, Fig. 4 (f) shows the angular frequency generated by the controller. It is seen that the controller tracks the reference active and reactive power with zero steadystate error, and the rise time of the system is about $10 \mathrm{~ms}$, as expected.

Also, Fig. 5 shows the simulation results of the system upon a fault occurrence and phase jump. Initially, the converter injects $P=4 \mathrm{MW}$ and $Q=2 \mathrm{MVAR}$ into the grid. At $t=0.05 \mathrm{~s}$, a $100 \mathrm{~ms}$ three-phase fault that makes the grid voltage $0.2 \mathrm{pu}$ occurs in the system. It is seen that the injected power is recovered since based on the physical attributes of the system, the reference active power does not exceed the line power transmission limit. At $t=0.15 \mathrm{~s}$, the fault is cleared, and the grid voltage is recovered. It is seen that the controller copes with the fault clearance. Additionally, at $t=0.25 \mathrm{~s}$, 
(a)

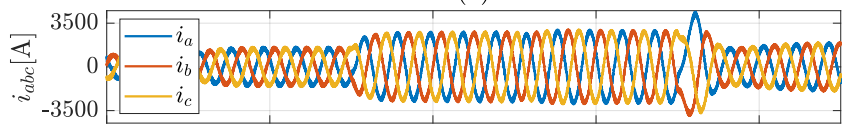

(b)

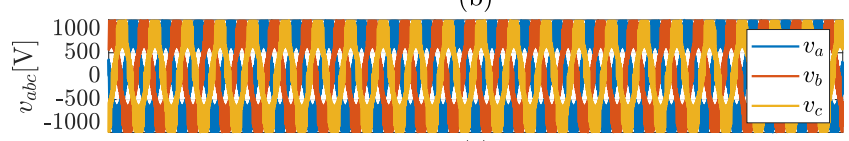

(c)

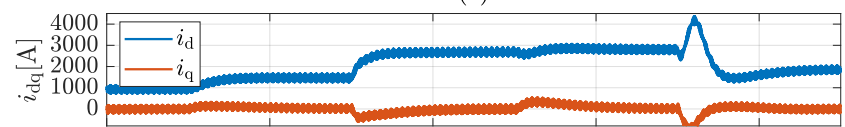

(d)

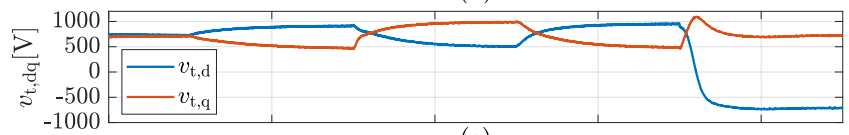

(e)

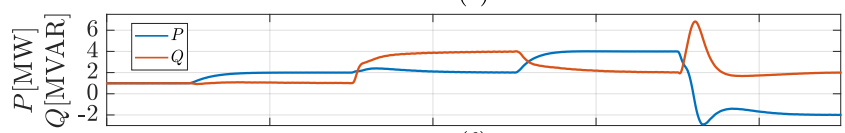

(f)

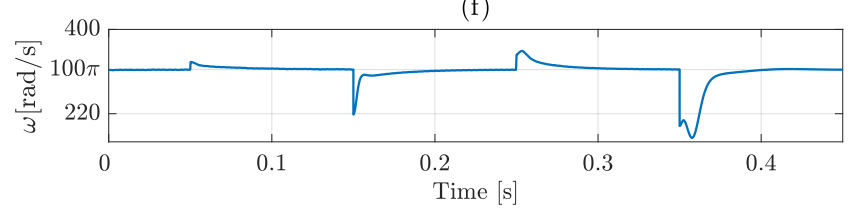

Fig. 4. The simulation results of the system with $\mathrm{SCR}=17$ upon various active and reactive power references change: a) three-phase grid currents $\left(i_{a b c}\right)$, b) the converter's filter's output three-phase line-to-neutral voltage, c) the dq-components of the grid current $\left.\left(i_{\mathrm{dq}}\right), \mathrm{d}\right)$ the dq-components of the terminal voltage $\left(v_{\mathrm{t}, \mathrm{dq}}\right)$, e) the injected active and reactive power into the grid, and f) the estimated angular frequency generated by the controller.

a $20^{\circ}$ phase jump occurs at the grid side voltage. It is seen that the system can maintain stability and injects the reference active and reactive powers into the grid after the phase jump occurrence.

2) Weak grid $(S C R=1.2)$ : In this part, the grid inductance and resistance are chosen as $L_{\mathrm{g}}=680 \mu \mathrm{H}$ and $R_{\mathrm{g}}=72 \mathrm{~m} \Omega$, respectively, making $\mathrm{SCR}=1.2$. Also, the PI controller employed in the current controller is $2500 \times \frac{770 \times 10^{-6} s+0.082}{s}$, making the current controller a first-order system with $\tau=0.0004 \mathrm{~s}$. Similar to the previous test, the $k_{p}$ is chosen equal to 100 , causing a $10 \mathrm{~ms}$ overall time constant. Initially, the converter injects $1 \mathrm{MW}$ and 1 MVAR of active and reactive power, respectively. At $t=0.05 \mathrm{~s}$, the active power set-point is changed to $2 \mathrm{MW}$. At $t=0.15 \mathrm{~s}$, the reactive power set-point steps up to 4 MVAR, while the active power remains unchanged. At $t=0.25 \mathrm{~s}$, the power factor is changed from 0.44 to 0.9 , changing the active and reactive power set-points to $4 \mathrm{MW}$ and $2 \mathrm{MVAR}$, respectively. Finally, at $t=0.35 \mathrm{~s}$, the converter's active power set-point is changed to $-2 \mathrm{MW}$, making the converter to work as a rectifier. Fig. 6 depicts the simulation results of this system. Fig. 6 (a) shows the three-phase output current, Fig. 6 (b) depicts the converter's filter's output line-to-neutral three-phase voltage, Fig. 6 (c), is d- and qcomponents of the converter's output current, Fig. 6 (d), is d- and q-components of the converter's terminal voltage, Fig. 6 (e) depicts the output active and reactive power, and finally, Fig. 6 (f) shows the angular frequency generated by the controller. It is seen that the controller tracks the reference active and reactive power with zero steady-state error, and the rise time of the system is about $10 \mathrm{~ms}$, as expected.

Also, Fig. 7 shows the simulation results of the system upon a fault occurrence and phase jump. Initially, the converter injects

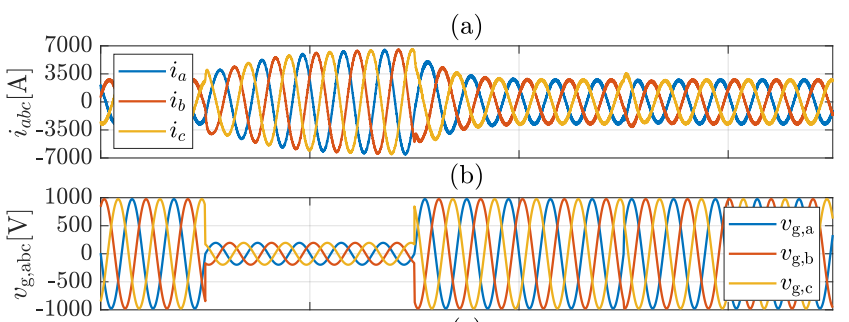

(c)

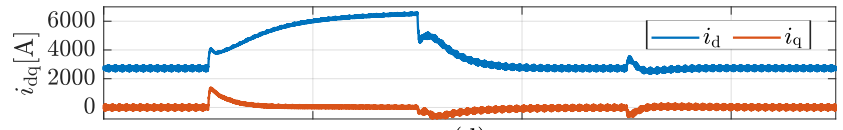

(d)

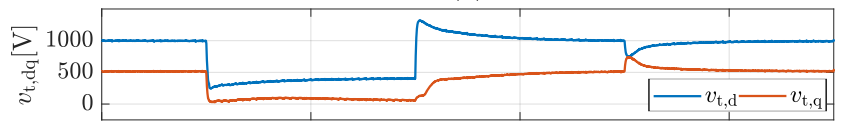

(e)

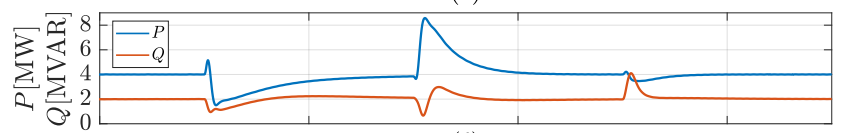

(f)

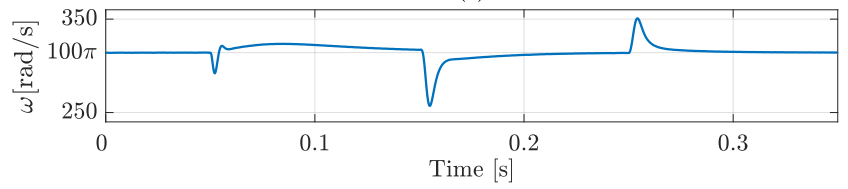

Fig. 5. The simulation results of the system with $\mathrm{SCR}=17$ upon a three-phase fault and grid voltage phase jump occurrence: a) three-phase grid currents $\left(i_{a b c}\right)$, b) the grid three-phase line-to-neutral voltage, c) the dq-components of the grid current $\left.\left(i_{\mathrm{dq}}\right), \mathrm{d}\right)$ the dq-components of the terminal voltage $\left(v_{\mathrm{t}, \mathrm{dq}}\right)$, e) the injected active and reactive power into the grid, and f) the estimated angular frequency generated by the controller.

$P=4 \mathrm{MW}$ and $Q=2 \mathrm{MVAR}$ into the grid. At $t=0.05 \mathrm{~s}$, a $100 \mathrm{~ms}$ three-phase fault that makes the grid voltage $0.2 \mathrm{pu}$ occurs in the system. However, it is seen that the system output power and generated angular frequency have a constant steady-state error during the fault. The reason is in (8), the controllers corresponding to $\Delta \omega$ are just proportional controllers. This prevents creating accumulative error in the generated frequency, and hence, the output active power. After the fault clearance $(t=0.15 \mathrm{~s})$, the line's power transferred limit is increased again, and the output active power is recovered. In addition, at $t=0.25 \mathrm{~s}$, a $20^{\circ}$ phase jump occurs at the grid side voltage. It is seen that the system can maintain stability and inject the reference active and reactive powers into the grid after the phase jump occurrence.

3) Comparative Analysis: To compare the performance of the proposed control design with the VMDPC while connected to a weak grid, similar active and reactive reference changes are applied to a system that has the VMDPC control structure. Using the VMDPC, the system is unable to work in a weak grid unless the gain of the system, and hence its speed, is decreased drastically. Fig. 8 shows the simulation results of the VMDPC. Similar to the tests applied to the proposed controller in this study, initially, the IBR injects $1 \mathrm{MW}$ and 1 MVAR. At $t=1 \mathrm{~s}$, the active power setpoint is changed to $2 \mathrm{MW}$. At $t=3.5 \mathrm{~s}$, the reactive power set-point steps up to 4 MVAR, while the active power remains unchanged. At $t=6 \mathrm{~s}$, the power factor is changed from 0.44 to 0.9 , changing the active and reactive power set-points to $4 \mathrm{MW}$ and 2 MVAR, respectively. Finally, at $t=8.5 \mathrm{~s}$, the converter's active power setpoint is changed to $-2 \mathrm{MW}$, making the converter a rectifier. It is seen that the rise time of the system is about $1 \mathrm{~s}$, which is 100 times slower than the controller proposed in this study. It should be 
(a)

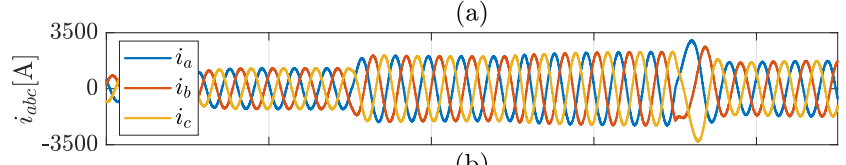

(b)

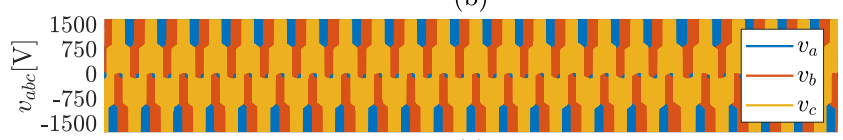

(c)

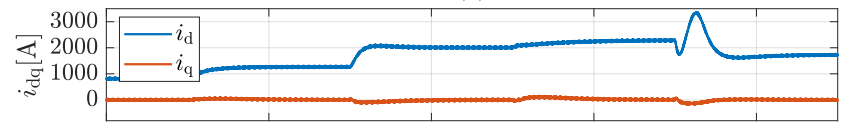

(d)

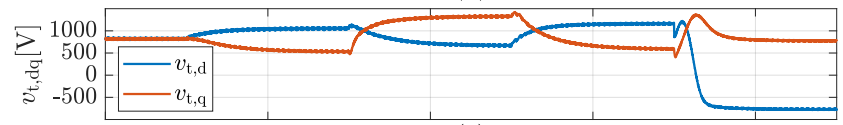

(e)

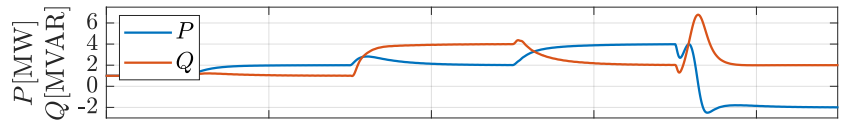

(f)

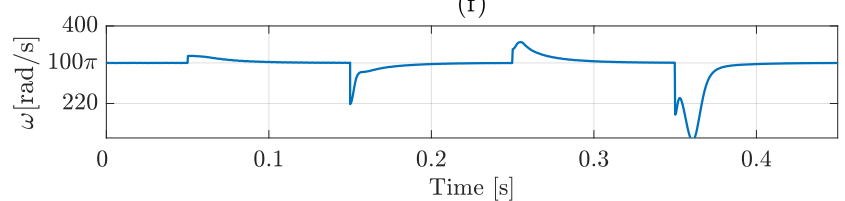

Fig. 6. The simulation results of the system with $\mathrm{SCR}=1.2$ upon various active and reactive power references change: a) three-phase grid currents $\left(i_{a b c}\right)$, b) the grid three-phase line-to-neutral voltage, c) the dq-components of the grid current $\left.\left(i_{\mathrm{dq}}\right), \mathrm{d}\right)$ the dq-components of the terminal voltage $\left(v_{\mathrm{t}, \mathrm{dq}}\right)$, e) the injected active and reactive power into the grid, and $\mathrm{f}$ ) the estimated angular frequency generated by the controller.

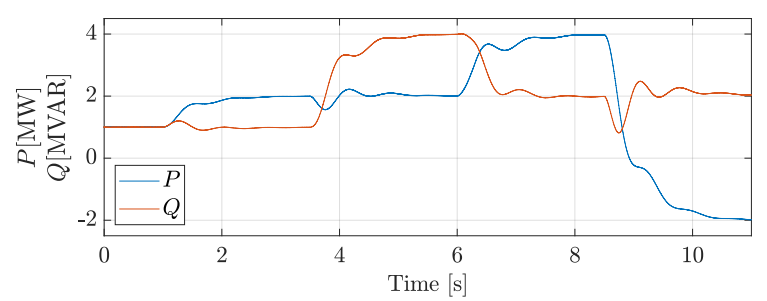

Fig. 8. The simulation results (active and reactive powers) of the VMDPC [34], when connected to a very weak grid with $\mathrm{SCR}=1.2$, upon active and reactive power references change.

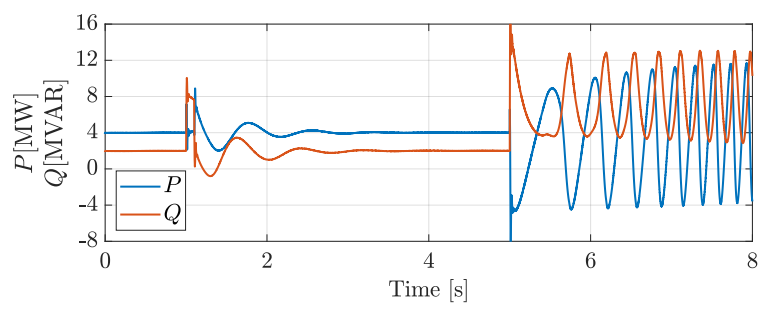

Fig. 9. The simulation results (active and reactive powers) of the VMDPC [34], when connected to a very weak grid with $\mathrm{SCR}=1.2$, upon a three-phase grid-side fault and $20^{\circ}$ grid voltage phase jump.

noted that, when connected to a weak grid, the VMDPC becomes unstable if the overall gain of the system increases (to make the system faster).

Additionally, a $100 \mathrm{~ms}$ three-phase grid-side fault that causes the grid voltage to drop to $0.2 \mathrm{pu}$ followed by a $20^{\circ}$ grid voltage phase jump is applied to the same system using the VMDPC connected to the same weak grid. The simulation result is presented in Fig. 9. At $t=1$, the three-phase fault takes place and gets clear after $100 \mathrm{~ms}$. It is seen that although the controller can stabilize the system, the settling time is about $1.5 \mathrm{~s}$, which is much slower than the (a)

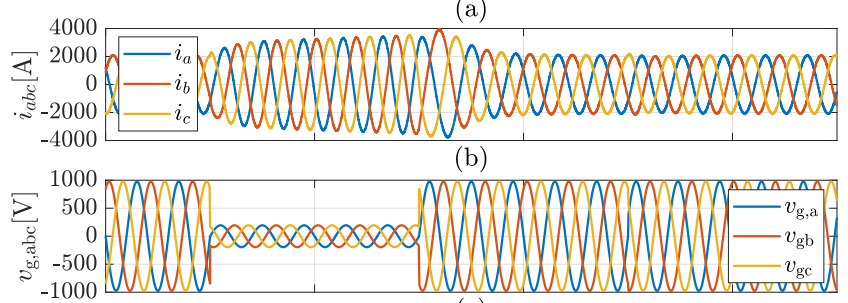

(c)

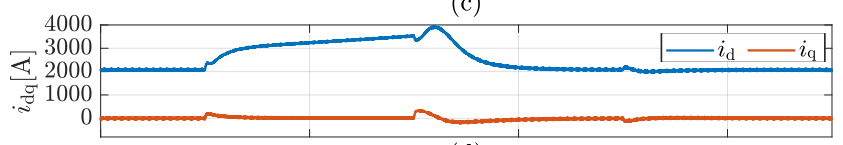

(d)

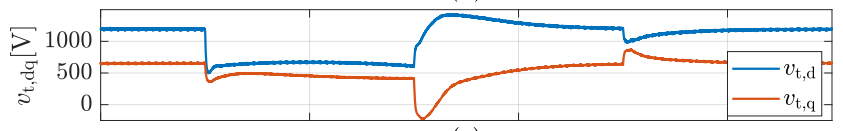

(e)

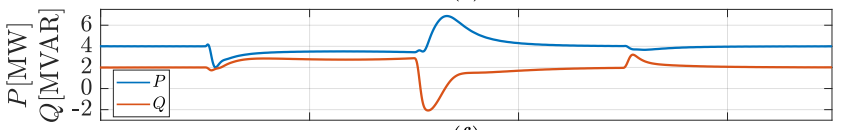

(f)

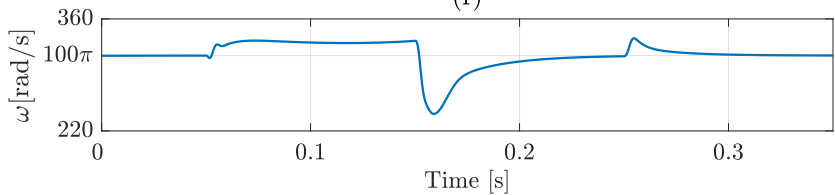

Fig. 7. The simulation results of the system with $\mathrm{SCR}=1.2$ upon a three-phase fault and grid voltage phase jump occurrence: a) three-phase grid currents $\left(i_{a b c}\right)$, b) the grid three-phase line-to-neutral voltage, c) the dq-components of the grid current $\left.\left(i_{\mathrm{dq}}\right), \mathrm{d}\right)$ the dq-components of the terminal voltage $\left(v_{\mathrm{t}, \mathrm{dq}}\right)$, e) the injected active and reactive power into the grid, and $f$ ) the estimated angular frequency generated by the controller.

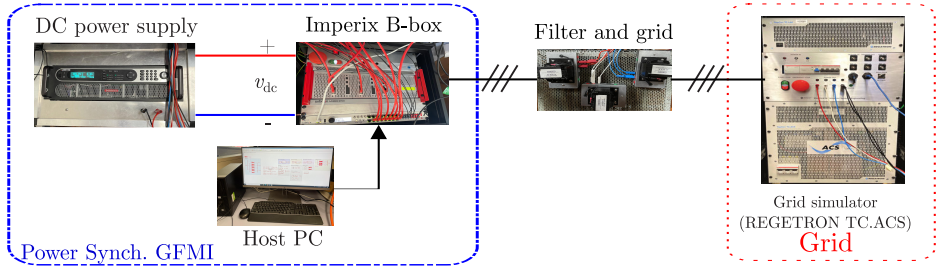

Fig. 10. The experimental setup.

TABLE II

THE PARAMETERS OF THE EXPERIMENTAL SETUP.

\begin{tabular}{c|c|c}
\hline \hline Quantity & Value & Comment \\
\hline \hline$L_{\mathrm{f}}$ & $14 \mathrm{mH}$ & Inverter Filter Inductance \\
$R_{\mathrm{f}}$ & $0.5 \Omega$ & Series Resistance of $L_{\mathrm{f}}$ \\
\hline$S_{\mathrm{base}}$ & $1 \mathrm{KVA}$ & Inverter Rated Power \\
$v_{\mathrm{g}}$ & $100 \mathrm{~V}$ & Grid Line-to-Line Voltage (rms) \\
$v_{\mathrm{dc}}$ & $300 \mathrm{~V}$ & DC Bus Voltage \\
\hline$f_{\mathrm{sw}}$ & $20 \mathrm{kHz}$ & PWM Carrier Frequency \\
$f$ & $50 \mathrm{~Hz}$ & System Nominal Frequency \\
$f_{\mathrm{filt}}$ & $200 \mathrm{~Hz}$ & Power Measurement LPF Bandwidth \\
\hline
\end{tabular}

proposed controller's performance. Additionally, at $t=5 \mathrm{~s}$, a $20^{\circ}$ phase jump occurs at the grid voltage. It is seen that, contrary to the introduced control method in this study, the VMDPC fails to stabilize the system upon the phase jump.

As a conclusion, the LPV controller proposed in this paper has a major advantage compared with the VMDPC while the system is connected to a weak grid. First, the proposed controller is quite faster compared with the VMDPC. Besides, it is seen that in case of sever disturbance occurrence, such as a phase jump, the VMDPC cannot stabilize the system while the proposed LPV controller used for the control structure in [38] rejects the disturbance properly. 
(a)

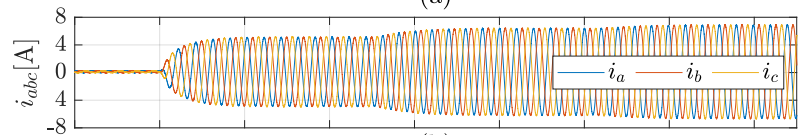

(b)

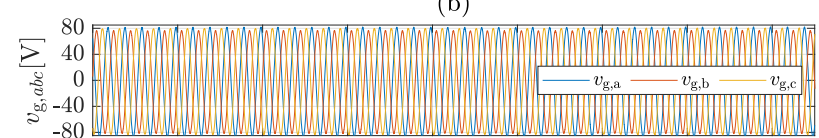

(c)

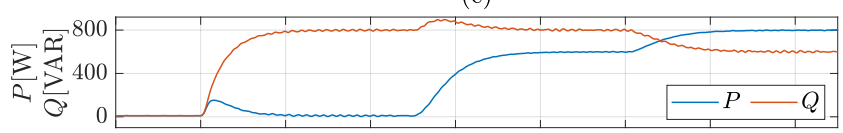

(d)

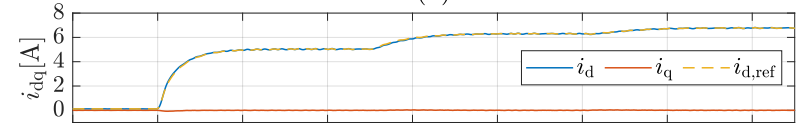

(e)

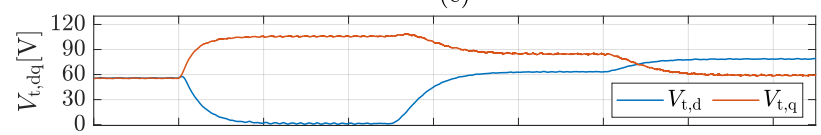

(f)

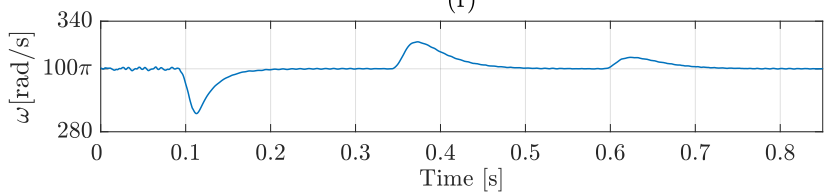

Fig. 11. The experimental results of the power synchronized GLFI while connected to a strong grid with $\mathrm{SCR}=31$ showing the zero-start, as well as active and reactive reference changes.

\section{B. Experimental Results}

To experimentally evaluate the performance of the proposed controller and to validate the simulation results, an experimental platform based on Imperix B-Box and Regatron AC Power Supply is designed, which is shown in Fig. 10. The corresponding control structures are implemented in Matlab/Simulink, and the control interface is done via Imperix ACG BB Control software. Table II shows the system's parameters. Also, the inner current controller's gain is set to 2500 . Additionally, the parameter $k_{p}$ is set to 25 in all tests, making the rise time of the system equal to $40 \mathrm{~ms}$. In these tests, the performance of the controller for active, reactive and power factor changes is evaluated.

In the first test, the grid inductance and resistance are set to $1 \mathrm{mH}$ and $0.03 \Omega$, respectively, making the $\mathrm{SCR}=31$. Initially, the inverter injects no active or reactive power into the grid. At $t=0.1 \mathrm{~s}$, the reactive power reference is changed to $800 \mathrm{VAR}$. At $t=0.35 \mathrm{~s}$, the active power set-point is changed to $600 \mathrm{~W}$. Finally, at $t=0.6 \mathrm{~s}$, the power factor is changed from 0.6 to 0.8 , while the apparent power is kept at 1 pu. Fig. 11 shows the experimental results of this test. Fig. 11(a) is the threephase injected currents, Fig. 11(b) is the three-phase grid line-toneutral voltages, Fig. 11(c) shows the active and reactive powers, Fig. 11(d) depicts the $\mathrm{d}$ and q-components of the injected current, as well as the generated $i_{\mathrm{d}}$ reference value by the controller, Fig. 11(e) depicts the $d$ and q-components of the terminal voltage, and finally, Fig. 11(f) shows the angular frequency estimated by the controller. It is seen that the system's rise time is about $40 \mathrm{~ms}$, and the controller regulates the $P$ and $Q$ upon changes in their references.

In the second test, the grid inductance and resistance are set to $16 \mathrm{mH}$ and $0.53 \Omega$, respectively, making the $\mathrm{SCR}=1.9$. Initially, the inverter injects no active or reactive powers into the grid. At $t=0.1 \mathrm{~s}$, the reactive power reference is changed to 800 VAR. (a)

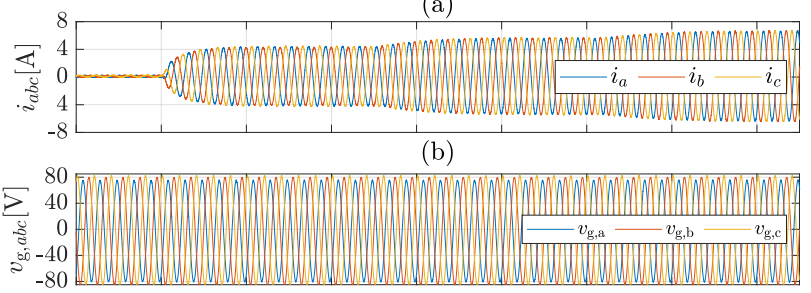

(c)

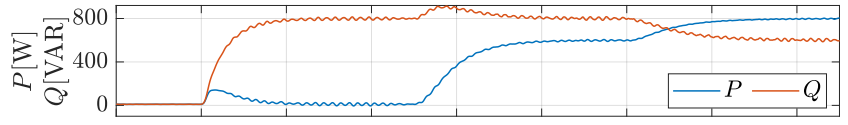

(d)

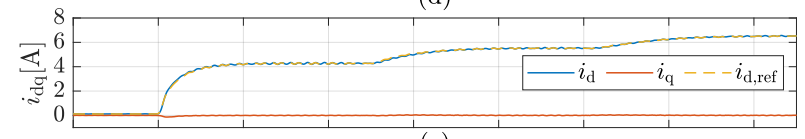

(e)
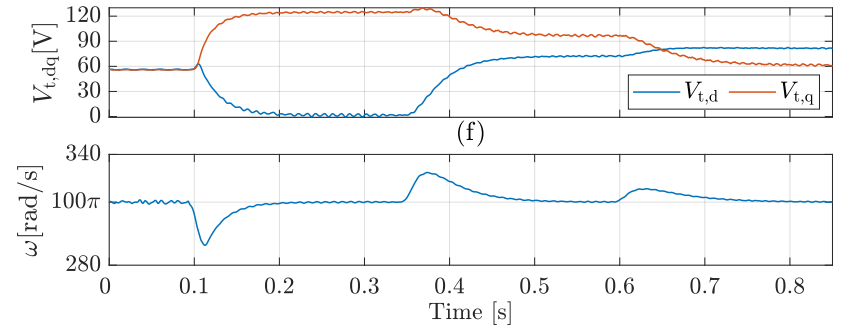

Fig. 12. The experimental results of the power synchronized GFLI while connected to a weak grid with $\mathrm{SCR}=1.9$ showing the zero-start, as well as active and reactive reference changes.

At $t=0.35 \mathrm{~s}$, the active power set-point is changed to $600 \mathrm{~W}$. Finally, at $t=0.6 \mathrm{~s}$, the power factor is changed from 0.6 to 0.8 , while the apparent power is kept at 1 pu. Fig. 12 shows the experimental results of this test. Fig. 12(a) is the three-phase injected currents, Fig. 12(b) is the three-phase grid line-to-neutral voltages, Fig. 12(c) shows the active and reactive power, Fig. 12(d) depicts the $\mathrm{d}$ and q-components of the injected current, as well as the generated $i_{\mathrm{d}}$ reference value by the controller, Fig. 11(e) depicts the $\mathrm{d}$ and q-components of the terminal voltage, and finally, Fig. 12(f) shows the angular frequency estimated by the controller. It is seen that the system's rise time is about $40 \mathrm{~ms}$, and the controller regulates the $P$ and $Q$ upon changes in their references.

Based on the results shown in the simulation and experimental tests, it is seen that the proposed controller rectifies the issues present in [38] control design method, i.e., has a constant time constant for different operating points and can perform as a rectifier with no need to modify the controller. Besides, contrary to the proposed method in [38], the proposed controller has a parametric, easy-to-design structure.

It is worth mentioning that various experimental tests to verify the ability of the system in coping with a $20^{\circ}$ phase jump and grid fault are done, which could not be put in the manuscript due to the length issue and could be provided in the second review.

\section{Conclusions}

This paper proposes an LPV loop-shaping controller for powersynchronized PLL-less GFLIs. IBRs enjoying this structure do not have any stability issues while connected to very strong or very weak grids; also, it is shown that using this controller, the performance of the system does not rely on the operating point, i.e., it varies its gains such that it maintains a constant rise time while working in different operating points. Additionally, the designed controller allows the system to work as a bi-directional converter. Besides, the proposed controller shapes the system's 
open-loop transfer function such that IBR's output active and reactive power are decoupled, and the rise time of the system can easily be tuned by changing the system's open-loop gain. Moreover, it is mathematically shown that the system is robust despite discrepancies that might occur in grid inductance and resistance estimation. Finally, The performance of the proposed control structure is validated via simulation and in experiment.

\section{REFERENCES}

[1] K. E. et al., Connection of Wind Farms to Weak AC Networks. WG B4.62.671, CIGRE, 2016.

[2] F. Blaabjerg, R. Teodorescu, M. Liserre, and A. V. Timbus, "Overview of control and grid synchronization for distributed power generation systems," IEEE Transactions on Industrial Electronics, vol. 53, no. 5, pp. 1398-1409, 2006.

[3] R. H. Lasseter, Z. Chen, and D. Pattabiraman, "Grid-forming inverters: A critical asset for the power grid," IEEE Journal of Emerging and Selected Topics in Power Electronics, vol. 8, no. 2, pp. 925-935, 2020.

[4] T. Xia, X. Zhang, G. Tan, and Y. Liu, "All-pass-filter-based pll for singlephase grid-connected converters under distorted grid conditions," IEEE Access, vol. 8, pp. 106226-106233, 2020.

[5] A. Luna, J. Rocabert, J. I. Candela, J. R. Hermoso, R. Teodorescu, F. Blaabjerg, and P. Rodríguez, "Grid voltage synchronization for distributed generation systems under grid fault conditions," IEEE Transactions on Industry Applications, vol. 51, no. 4, pp. 3414-3425, 2015.

[6] B. Bahrani, S. Kenzelmann, and A. Rufer, "Multivariable-pi-based $d q$ current control of voltage source converters with superior axis decoupling capability," IEEE Transactions on Industrial Electronics, vol. 58, no. 7, pp. 3016-3026, 2010.

[7] B. Bahrani, M. Vasiladiotis, and A. Rufer, "High-order vector control of gridconnected voltage-source converters with lcl-filters," IEEE Transactions on Industrial Electronics, vol. 61, no. 6, pp. 2767-2775, 2014.

[8] X. Wang, M. G. Taul, H. Wu, Y. Liao, F. Blaabjerg, and L. Harnefors, "GridSynchronization Stability of Converter-Based Resources-An Overview," IEEE Open Journal of Industry Applications, vol. 1, pp. 115-134, 2020.

[9] J. Machowski, Z. Lubosny, J. W. Bialek, and J. R. Bumby, Power system dynamics: stability and control. John Wiley \& Sons, 2020.

[10] L. Zhang, L. Harnefors, and H.-P. Nee, "Power-synchronization control of grid-connected voltage-source converters," IEEE Transactions on Power systems, vol. 25, no. 2, pp. 809-820, 2009.

[11] X. Meng, J. Liu, and Z. Liu, "A generalized droop control for grid-supporting inverter based on comparison between traditional droop control and virtual synchronous generator control," IEEE Transactions on Power Electronics, vol. 34, no. 6, pp. 5416-5438, 2019.

[12] $\mathrm{H}$. Wu and $\mathrm{X}$. Wang, "Design-oriented transient stability analysis of pllsynchronized voltage-source converters," IEEE Transactions on Power Electronics, vol. 35, no. 4, pp. 3573-3589, 2020.

[13] X. Wang and F. Blaabjerg, "Harmonic stability in power electronic-based power systems: Concept, modeling, and analysis," IEEE Transactions on Smart Grid, vol. 10, no. 3, pp. 2858-2870, 2019.

[14] M. G. Taul, X. Wang, P. Davari, and F. Blaabjerg, "Systematic approach for transient stability evaluation of grid-tied converters during power system faults," in IEEE Energy Conversion Congress and Exposition (ECCE), 2019, pp. 5191-5198.

[15] J. Zhao, M. Huang, H. Yan, C. K. Tse, and X. Zha, "Nonlinear and transient stability analysis of phase-locked loops in grid-connected converters," IEEE Transactions on Power Electronics, vol. 36, no. 1, pp. 1018-1029, 2021.

[16] H. Wu and X. Wang, "Transient angle stability analysis of grid-connected converters with the first-order active power loop," in Applied Power Electronics Conference and Exposition (APEC). IEEE, 2018, pp. 3011-3016.

[17] H. Geng, L. Liu, and R. Li, "Synchronization and Reactive Current Support of PMSG-based Wind Farm during Severe Grid Fault," IEEE Transactions on Sustainable Energy, vol. 9, no. 4, pp. 1596-1604, 2018.

[18] F. Andrade, K. Kampouropoulos, L. Romeral, J. C. Vasquez, and J. M. Guerrero, "Study of large-signal stability of an inverter-based generator using a lyapunov function," in IECON 2014 - 40th Annual Conference of the IEEE Industrial Electronics Society, 2014, pp. 1840-1846.

[19] M. Z. Mansour, S. P. Me, S. Hadavi, B. Badrazadeh, A. Karimi, and B. Bahrani, "Nonlinear transient stability analysis of phased-locked loop based grid-following voltage source converters using lyapunov's direct method," IEEE Journal of Emerging and Selected Topics in Power Electronics, 2021.

[20] Y. Liao, X. Wang, F. Liu, K. Xin, and Y. Liu, "Sub-synchronous control interaction in grid-forming vscs with droop control," in 2019 4th IEEE Workshop on the Electronic Grid (eGRID). IEEE, 2019, pp. 1-6.
[21] S. Wang, Z. Liu, J. Liu, D. Boroyevich, and R. Burgos, "Small-signal modeling and stability prediction of parallel droop-controlled inverters based on terminal characteristics of individual inverters," IEEE Transactions on Power Electronics, vol. 35, no. 1, pp. 1045-1063, 2019.

[22] $\mathrm{H}$. Wu and $\mathrm{X}$. Wang, "Design-oriented transient stability analysis of gridconnected converters with power synchronization control," IEEE Transactions on Industrial Electronics, vol. 66, no. 8, pp. 6473-6482, 2019.

[23] D. Pan, X. Wang, F. Liu, and R. Shi, "Transient stability of voltage-source converters with grid-forming control: A design-oriented study," IEEE Journal of Emerging and Selected Topics in Power Electronics, vol. 8, no. 2, pp. 1019-1033, 2019.

[24] G. Denis, T. Prevost, P. Panciatici, X. Kestelyn, F. Colas, and X. Guillaud, "Improving robustness against grid stiffness, with internal control of an ac voltage-controlled vsc." in 2016 IEEE Power and Energy Society General Meeting (PESGM). IEEE, 2016.

[25] G. Li, Y. Chen, A. Luo, Z. He, H. Wang, Z. Zhu, W. Wu, and L. Zhou, "Analysis and mitigation of subsynchronous resonance in series-compensated grid-connected system controlled by a virtual synchronous generator," IEEE Transactions on Power Electronics, vol. 35, no. 10, pp. 11 096-11 107, 2020.

[26] M. G. Taul, X. Wang, P. Davari, and F. Blaabjerg, "Current limiting control with enhanced dynamics of grid-forming converters during fault conditions," IEEE Journal of Emerging and Selected Topics in Power Electronics, vol. 8, no. 2, pp. 1062-1073, 2019.

[27] M. Malinowski, M. Jasinski, and M. P. Kazmierkowski, "Simple direct power control of three-phase pwm rectifier using space-vector modulation (dpcsvm)," IEEE Transactions on Industrial Electronics, vol. 51, no. 2, pp. 447454, 2004.

[28] T. Noguchi, H. Tomiki, S. Kondo, and I. Takahashi, "Direct power control of pwm converter without power-source voltage sensors," IEEE transactions on industry applications, vol. 34, no. 3, pp. 473-479, 1998.

[29] A. Bouafia, J. Gaubert, and F. Krim, "Predictive direct power control of threephase pulsewidth modulation (pwm) rectifier using space-vector modulation (svm)," IEEE Transactions on Power Electronics, vol. 25, no. 1, pp. 228-236, 2010.

[30] D. Zhi and L. Xu, "Direct power control of dfig with constant switching frequency and improved transient performance," IEEE Transactions on Energy Conversion, vol. 22, no. 1, pp. 110-118, 2007.

[31] S. Vazquez, J. A. Sanchez, J. M. Carrasco, J. I. Leon, and E. Galvan, "A model-based direct power control for three-phase power converters," IEEE Transactions on Industrial Electronics, vol. 55, no. 4, pp. 1647-1657, 2008.

[32] J. Hu, L. Shang, Y. He, and Z. Zhu, "Direct active and reactive power regulation of grid-connected dc/ac converters using sliding mode control approach," IEEE transactions on power electronics, vol. 26, no. 1, pp. 210 $222,2010$.

[33] Y. Gui, G. H. Lee, C. Kim, and C. C. Chung, "Direct power control of grid connected voltage source inverters using port-controlled hamiltonian system," International Journal of Control, Automation and Systems, vol. 15, no. 5, pp. 2053-2062, 2017.

[34] Y. Gui, X. Wang, F. Blaabjerg, and D. Pan, "Control of grid-connected voltage-source converters: The relationship between direct-power control and vector-current control," IEEE Industrial Electronics Magazine, vol. 13, no. 2, pp. 31-40, 2019.

[35] Y. Gui, X. Wang, and F. Blaabjerg, "Vector current control derived from direct power control for grid-connected inverters," IEEE Transactions on Power Electronics, vol. 34, no. 9, pp. 9224-9235, 2019.

[36] Y. Gui, X. Wang, H. Wu, and F. Blaabjerg, "Voltage-modulated direct power control for a weak grid-connected voltage source inverters," IEEE Transactions on Power Electronics, vol. 34, no. 11, pp. 11 383-11 395, 2019.

[37] S. Milad Hoseinizadeh, S. Ouni, H. Karimi, M. Karimi-Ghartemani, and K. L. Lian, "Comparison of PLL-Based and PLL-Less vector current controllers," IEEE Journal of Emerging and Selected Topics in Power Electronics, 2021.

[38] B. Bahrani, "Power-synchronized grid following inverter without a Phaselocked Loop," IEEE Access, 2021.

[39] J. Mohammadpour and C. W. Scherer, Control of linear parameter varying systems with applications. Springer Science \& Business Media, 2012.

[40] J. H. Suárez, H. M. Gomes, A. J. Sguarezi Filho, D. A. Fernandes, and F. F. Costa, "Grid impedance estimation for grid-tie inverters based on positive sequence estimator and morphological filter," Electrical Engineering, 2020.

[41] N. Mohammed, M. Ciobotaru, and G. Town, "Fundamental grid impedance estimation using grid-connected inverters: a comparison of two frequencybased estimation techniques," IET Power Electronics, vol. 13, no. 13, pp. 2730-2741, 2020. 